\title{
Quelques réflexions sur la fabrique de l'action enseignante dans une perspective diachronique
}

\section{Lin XUE}

\section{OpenEdition}

\section{Journals}

Édition électronique

URL : http://journals.openedition.org/rdlc/766

DOI : $10.4000 /$ rdlc.766

ISSN : 1958-5772

Éditeur

ACEDLE

Référence électronique

Lin XUE, «Quelques réflexions sur la fabrique de l'action enseignante dans une perspective

diachronique ", Recherches en didactique des langues et des cultures [En ligne], 12-2 | 2015, mis en ligne le 20 novembre 2015, consulté le 19 avril 2019. URL : http://journals.openedition.org/rdlc/766 ; DOI :

$10.4000 /$ rdlc.766

Ce document a été généré automatiquement le 19 avril 2019

\section{cc) $(\mathrm{T})(\mathrm{\Theta}$}

Recherches en didactique des langues et des cultures is licensed under a Creative Commons AttributionNonCommercial-NoDerivatives 4.0 International License 


\title{
Quelques réflexions sur la fabrique de l'action enseignante dans une perspective diachronique
}

\author{
Lin Xue
}

\section{Introduction}

1 Dans la littérature sur la didactique des langues et dans celle sur les sciences de l'éducation, les travaux sur l'enseignant, acteur-clé dans l'enseignement/apprentissage, constituent un pôle important (Borg, 2003, 2006). On y trouve notamment des recherches sur la pensée enseignante et sur l'agir professoral, sur la formation des enseignants et sur le teacher development. Si dans la notion même de teacher development se dégage l'idée de l'évolution, Mann, avec la révision des recherches antérieures sur ce sujet, souligne que

Language teacher development [...] is a continuing process of becoming and can never be finished; [...] a process of articulating an inner world of conscious choices made in response to the outer world of the teaching context [...] (2005: 105).

En admettant que l'interaction entre l'enseignant et des éléments extérieurs fasse évoluer sa pensée et ses pratiques, nous nous demandons quels pourraient être, chez un enseignant donné, les éléments concourant potentiellement à cette évolution et comment elle évolue. Le présent article s'intéresse ainsi à l'interprétation de la notion de fabrique de l'action enseignante dans une perspective diachronique. Le terme diachronique implique un suivi à long terme, il signifie en l'occurrence la mise en place d'un dispositif d'observation et d'entretiens durant un semestre afin de suivre un enseignant participant. Les commentaires de l'enseignant sur ses pratiques, produits tout au long du semestre, permettraient ainsi de voir de quelles manières il perçoit, observe et interprète ses actions, notamment la formation et l'évolution de son agir professoral. En d'autres termes, il s'agit d'observer comment la fabrique de l'action enseignante se concrétise dans les représentations de l'enseignant. Dans cet article, avec des exemples d'illustration, dont des verbalisations de deux enseignants débutants issues d'entretiens 
d'auto-confrontation ${ }^{1}$, nous essayerons, en reconstruisant l'évolution de leur agir professoral au cours d'un semestre à travers leurs auto-commentaires, de voir les différentes façons possibles de fabrication et d'évolution de leurs pratiques enseignantes.

\section{Corpus}

Les verbalisations des deux enseignants participants - un enseignant de chinois langue étrangère dans une université française à un public francophone et une enseignante de français langue étrangère dans une institution parisienne à un public sinophone - sont issues d'EAC (Entretiens d'Auto-Confrontation) simples, entretiens pendant lesquels l'enseignant est confronté à ses propres images vidéoscopiques (Clot et al., 2001: 4). Afin de reconstruire l'évolution de leurs pratiques enseignantes, nous les avons suivis pendant un semestre par l'observation d'un cours hebdomadaire de deux heures et différents entretiens dont l'EAC fait partie. En revenant sur ses propres actions lors du visionnage des séances filmées au début, au milieu et en fin de semestre, chacun des deux enseignants est invité à donner du sens à ses actes. Il faut également signaler que, dans les deux cas, il s'agit d'entretiens différés et tous les commentaires des enseignants relèvent de discours auto-sélectionnés, l'enquêtrice n'intervenant que pour obtenir davantage de détails sur les idées des enseignants.

Pour chacune des séances filmées, le choix des séquences, dont la durée totale est environ de trente à quarante minutes, s'effectue selon plusieurs principes. Le début et la fin du cours sont systématiquement pris en considération tandis que la durée des extraits choisis varie en fonction des séances. Au moins une activité complète est sélectionnée et il s'agit souvent de l'activité principale de la séance en question. En ce qui concerne d'autres activités, elles apparaissent plutôt sous forme de courts extraits fragmentaires qui peuvent en quelque sorte les représenter. Des scènes "propres" à la séance en question, c'est-à-dire des moments où nous constatons, par exemple, de fortes émotions (rire de l'enseignant ou des apprenants) ou de possibles difficultés de l'enseignant, sont prises en compte. Il s'agit d'habitude d'éléments nouveaux et occasionnels assez "saillants" par rapport aux séances précédentes.

5 L'ensemble des entretiens transcrits constitue notre corpus et, pour en faciliter la compréhension, le discours de l'enseignant sinophone cité ici est traduit en français.

6 En ce qui concerne l'analyse des données, aussi bien l'analyse de contenu que l'analyse du discours sont convoquées. Les verbalisations de l'enseignante sont d'abord codées et classifiées en fonction des macro-thématiques, enseignant, apprenant et contexte pour qu'ensuite, dans chacune des macro-catégories, les discours soient regroupés à partir de thèmes encore plus précis et forment des micro-groupes. Il faut préciser qu'une seule séquence ou même un seul énoncé peut posséder plusieurs codes différents. Un exemple peut sans douter l'expliquer mieux :

\section{$\{00: 52: 03\} 0150$ Enseignante}

encore une fois c'est très américain cette méthode + fin je m'inspire de de voilà les présentations $\uparrow+$ euh euh euh parler en public $\uparrow+$ parler en face d'une classe $\uparrow+$ apprendre à parler un peu fort $\uparrow+$ à utiliser les gestes $\uparrow+$ à utiliser la voix euh : je je je m'inspire beaucoup parce que j'ai euh + moi-même + euh appris les langues comme ça + avec ces méthodes-là+ donc bon + ça me plaisait PLUS + quand j'étais euh étu fin élève quand on faisait des jeux et quand on faisait semblant et que il y a de vraies 
situations de communication qui peuvent exister + je je je me prenais au jeu et je cherchais plus que si c'était un exercice de grammaire [...]

7 L'extrait ci-dessus, centré sur l'enseignante elle-même, se situe dans la macro-catégorie enseignant. Les énoncés soulignés sont ensuite regroupés dans la micro-catégorie méthodologie d'enseignement tandis que la séquence ci-après est plutôt centrée sur l'expérience d'apprentissage : l'enseignante nous raconte ses expériences avec la méthode " américaine" pendant sa scolarisation.

Figure 1 - catégorisation du corpus

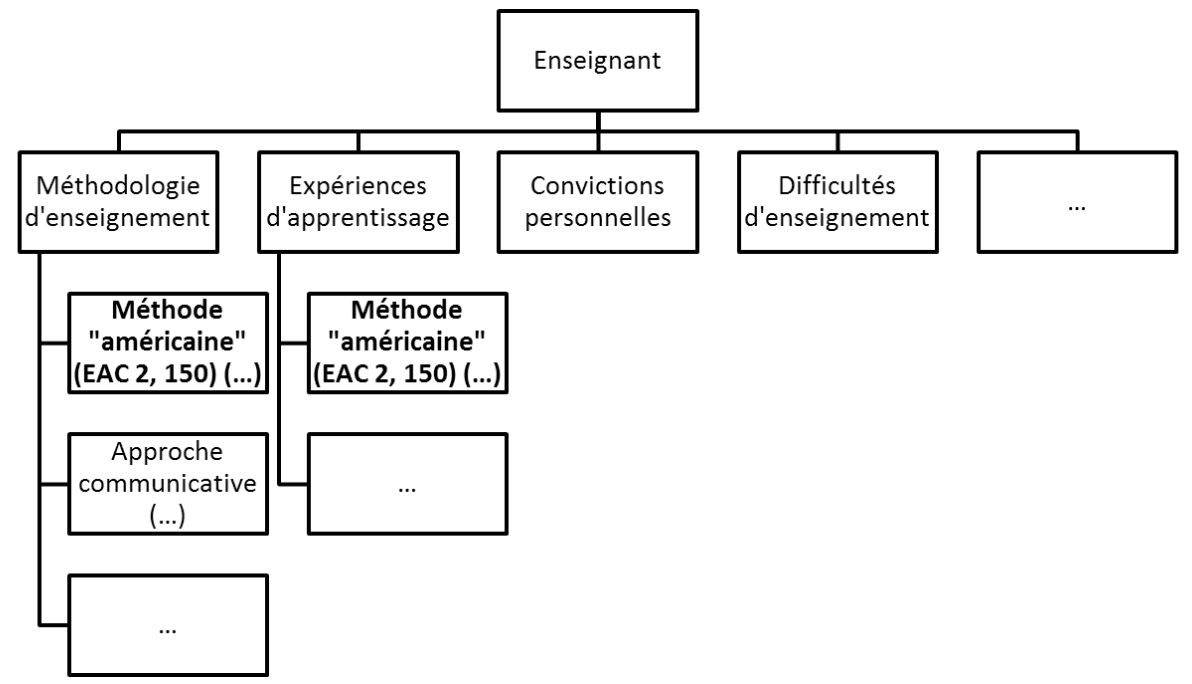

8 Une fois catégorisés, les discours portant sur le même thème sont analysés à partir des entrées discursives, comme par exemple, les paradigmes désignationnels (Mortureux, 1993) dont les substitutions lexicales peuvent donner des pistes sur le changement des représentations de l'énonciateur. Des marques énonciatives méritent aussi d'être analysées: des pronoms personnels, inscrivant le locuteur dans le discours, nous renseignent sur sa relation avec l'énoncé en question et avec d'autres protagonistes mentionnés (Maingueneau, 1994 : 22). Des éléments modaux ${ }^{2}$ qui dégagent la subjectivité discursive permettent de situer la verbalisation de l'enseignant sur le plan logique, appréciatif et évaluatif. Toujours par rapport à l'extrait ci-dessus, l'engagement personnel de l'enseignante se manifeste par la récurrence du "je" et son appréciation de la méthode "américaine" par les éléments modaux tels que "beaucoup", "plaisait" et "plus" (cf. 3.).

9 S'effectue par la suite la mise en relation des résultats d'analyse. Ceux des extraits portant sur des thèmes identiques ou similaires sont à croiser, ce qui permet de voir non seulement les représentations de l'enseignant sur un élément donné à différents moments et ainsi leur évolution, mais aussi les éventuels éléments participant à la formation et à l'évolution de l'action enseignante.

\section{Apprenticeship of observation et l'anti-fabrique de l'action enseignante}

10 Le mot évolution sous-entend dans un premier temps l'instabilité. Cette instabilité peut être repérée en début de carrière quand l'enseignant novice tâtonne et tente, découvre et 
réessaie; elle pourrait également s'expliquer par la vocation professionnelle d'un enseignant expérimenté qui s'initie aux Tice (Technologies de l'Information et de la Communication pour l'Éducation) pour actualiser ses méthodologies d'enseignement. La fabrique de l'action enseignante est ainsi en premier lieu un processus pendant lequel l'enseignant construit, déconstruit et reconstruit sa pensée et ses actions enseignantes. Dans les cas de déconstruction, l'enseignant mettrait en place une sorte d'anti-fabrique qui, en raison d'un fort contraste entre ses convictions ou habitudes existantes et les informations nouvellement arrivées, se traduit par un rejet de ces dernières.

11 La fabrique de l'action enseignante ne se construit cependant pas à partir du vide. S'il y a des traces d'évolution, cela implique qu'un embryon existait au préalable. Les deux enseignants novices participants mentionnent sans arrêt leurs propres expériences d'apprentissage de langues, ce qui correspond au concept "apprenticeship of observation" en littérature anglo-saxonne: l'apprenant regarde le devant de la scène comme un spectateur qui assiste à une pièce de théâtre (Lortie, 1975:61-62). C'est-à-dire que tout acteur socialisé et scolarisé, quel que soit son métier, possède des représentations sur le fonctionnement de l'enseignement/apprentissage issues de ses expériences. En ce sens, la fabrique de l'action enseignante commence dès le jeune âge, par l'observation des cadres socio-scolaires dont l'enseignant fait partie quand il est lui-même apprenant.

Si l'enseignante française corrige rarement à l'oral, c'est en raison de l'expérience désagréable vécue après son retour en France métropolitaine - elle se faisait reprendre " violemment" en raison de son accent africain :

\section{$\{01: 10: 37\} 0401$ Enseignante}

si les étudiants aiment bien le professeur $\uparrow$ et ils ont pas peur de faire des fautes+ bon c'est gagné + [...] tu sais + franchement bon + peut-être t'as déjà vécu cette situation mais + en France $\uparrow$ + moi quand chuis rentrée d'Afrique et que chuis chuis arrivée en France j'étais en Afrique fin en Côte d'Ivoire là où j'ai grandi $\uparrow+$ on parle pas le français euh exactement euh fin c'est une variation du français + et quand je suis arrivée en France j'étais en hypercorrection + constamment + on me faisait répéter et pourtant chuis française ah $\uparrow+$ on me faisait répéter CONSTAMMENT + reprendre mes phrases CONSTAMMENT + et ton accent : $\uparrow+$ tu viens d'où euh $\uparrow+$ tu vois de petites traces comme ça qui qui montraient que + que les gens avaient bien compris que j'étais pas ici ++ et les Français c'est vrai qu'en général ils aiment BIEN questionner sur l'accent $+[\ldots]$ donc les Français sont pas très accueillants quoi + DONC bon en classe si c'est DUR aussi euh $\uparrow$ et c'est DUR partout euh $\uparrow+$ il faut quand même leur donner envie d'apprendre cette langue $\uparrow+[\ldots]$

13 Les éléments modaux tels que "constamment" et "dur", répétés et accentués par l'enseignante, montrent à tel point cette expérience désagréable l'a marquée de telle façon qu'elle est décidée à ne pas employer ce mode de correction dans son enseignement. Si l'enseignante française construit son action en rejetant ce qui lui déplaisait lors de son apprentissage, elle reprend également, même de façon systématique, ce qui lui plaisait :

\section{$\{00: 52: 03\} 0150$ Enseignante}

encore une fois c'est très américain cette méthode + fin je m'inspire de de voilà les présentations $\uparrow+$ euh euh euh parler en public $\uparrow+$ parler en face d'une classe $\uparrow+$ apprendre à parler un peu fort $\uparrow+$ à utiliser les gestes $\uparrow+$ à utiliser la voix euh : je je je m'inspire beaucoup parce que j'ai euh + moi- 
même + euh appris les langues comme ça + avec ces méthodes-là + donc bon + ça me plaisait PLUS + quand j'étais euh étu fin élève quand on faisait des jeux et quand on faisait semblant et que il $\mathrm{y}$ a de vraies situations de communication qui peuvent exister + je je je me prenais au jeu et je cherchais plus que si c'était un exercice de grammaire euh bon même si c'est nécessaire hein je dis pas que c'est pas nécessaire [...]

14 Nous pouvons constater que l'enseignante française n'a pas hésité à mettre en place des méthodes qu'elle qualifie d'américaines, méthodes qui lui convenaient pendant son apprentissage des langues étrangères. Ce phénomène peut également être repéré chez l'enseignant sinophone qui, ayant particulièrement apprécié la méthodologie employée par un de ses enseignants de français - enseigner la langue cible en langue cible - préfère enseigner le chinois en chinois :

\section{$\{00: 40: 28\} 0144$ Enseignant}

[...] mon professeur de français il savait parler chinois + mais lui en fait il insistait pour nous apprendre le français en français + je trouve + c'est-à-dire + tu peux + éviter d'être influencé par beaucoup de choses dans ta langue originale + euh + l'influence des conceptions bizarres + c'est-à-dire que ce soit de l'anglais du chinois ou autre + je pense que c'est bien + c'est bien pour les étudiants [...] Si j'avais pu insister pour enseigner le chinois + en chinois + en chinois + ça aurait été mieux [...] en fait j'avais essayé le semestre dernier + mais après il me semble qu'ils comprenaient pas du tout + c'est-à-dire + pas $\mathrm{du}$ tout $+[. .$.$] le semestre prochain +$ pour tous les cours je vais essayer d'enseigner en chinois + je vais essayer

15 Malgré l'obstacle rencontré lors de son premier essai, l'enseignant chinois va tenter à nouveau l'expérience avec une approche du chinois en chinois, méthodologie pertinente selon ses expériences et ses convictions. Les exemples de cette sorte étant nombreux, il serait sans doute intéressant d'analyser comment une expérience mémorisée par un enseignant peut évoluer en composante de son répertoire didactique ${ }^{3}$. Ce qui est commun à ces exemples, c'est qu'il y a toujours un tri des outils ou des références didactiques, conscient ou inconscient, dans les scènes d'apprentissage dont l'enseignant est imprégné. Dans ce processus de sélection, le critère de filtrage tourne toujours autour de l'enseignant, de sa perception par rapport à son vécu.

Dans ces extraits, à partir des connecteurs logiques articulant les faits antérieurement vécus et les méthodologies actuellement mises en place, à partir des "je" et des "moi-même ", on peut voir que les enseignants sont bien conscients du rapport singulier entre leurs expériences d'apprentissage, les préférences personnelles et les pratiques d'enseignement. En ce sens, la fabrique de l'action enseignante suit un itinéraire historique dans lequel le passé pèse : les expériences d'apprentissage tracent le présent en passant par le ressenti personnel. Ainsi, ce processus de fabrique est aussi singulier chez chaque enseignant car il dépend beaucoup des traits personnels et des vécus, différents d'une personne à une autre.

17 L'influence des expériences vécues ne se situe pas seulement au niveau méthodologique, elle est également une des principales références à partir desquelles l'enseignant fait des hypothèses sur la psychologie et le comportement de ses propres étudiants. Il apparaît que pour ces deux enseignants, dans les cas où une ou plusieurs circonstance(s) du contexte présent correspondent à des expériences vécues par eux-mêmes, ils ont 
tendance à faire le rapprochement avec leur psychologie de l'époque - ressenti dans la mémoire suscitée par le contexte de celle de leurs étudiants.

\section{$\{00: 35$ :07\}0166 Enseignant}

[...] peut-être en fait ils ont même pas envie de parler + n'est-ce pas (rire de l'enquêtrice) comme moi avant je pense que quand j'apprenais des choses comme l'arabe + je ne préparais pas du tout + parce que je n'avais vraiment pas le temps à préparer j'écrivais ma thèse et n'avais vraiment pas le temps + quand j'y allais je pensais que je voulais pas vraiment m'entraîner + je m'y intéressais un peu + de toute façon + je venais écouter et puis + si je pouvais apprendre deux mots ou deux phrases tant mieux + c'était juste pour changer d'humeur + peut-être que les autres pensent aussi comme ça +

\section{$\{01: 19: 59\} 0225$ Enseignante}

[...] je sais que quand moi je suis valorisée par un professeur $\uparrow+\mathrm{j}$ 'ai envie de parler $\uparrow+$ j'ai je peux pas le décevoir $\uparrow+$ ça marche + l'affect ça marche + donc c'est ça que j'essaie de faire avec les étudiants + même s'il y a des choses à reprendre $+[. .$.$] les professeurs on sait souvent euh +$ ils sont avares de compliments ils sont radins + bon c'est difficile de d'être de vivre loin $\uparrow+$ de ses parents de sa famille à l'étranger d'entendre du français toute la journée $\uparrow+$ de de d'être dans une culture où on comprend rien euh $\uparrow+$ bon si on n'a pas au moins une personne qui nous dit que ce qu'on fait c'est bien $\uparrow++$ c'est trop difficile

\section{$\{01: 22$ :08\}0226 Enquêtrice}

c'est par rapport à tes propres expériences $\uparrow$

\section{$\{01: 22: 11\} 0227$ Enseignante}

ouais $\downarrow$ où j'étais à l'étranger $\uparrow+$ et j'étais pas dans des cultures euh si euh j'étais dans des cultures latines donc c'était proche quand même de ma culture $\uparrow+$ pourtant déjà c'était difficile $\uparrow+$ ma famille me manquait et tout + donc bon euh c'est pas facile ce qu'ils font + mettre en immersion comme ça euh pour six mois un an euh + vraiment j'ai de l'empathie et c'est pour ça que je pense que + bon peut-être c'est trop + (rire)

Ainsi, selon l'enseignant, beaucoup d'étudiants du cours du soir ne s'investissent pas beaucoup et cela est tout à fait compréhensible quand il rapproche la situation actuelle de son expérience de l'apprentissage de l'arabe, car la motivation du public coïncide en un sens avec la sienne, à cette époque-là. Et l'enseignante française, ayant vécu elle-même à l'étranger, se projette d'une certaine manière dans le rôle de ses étudiants et repense aux difficultés rencontrées pendant son séjour à l'étranger. Ainsi, elle donne systématiquement des compliments "exagérés" comme "bravo", "magnifique" et "excellent" pour que ces "pauvres", terme qu'elle emploie souvent pour désigner ses apprenants, se sentent valorisés. Le nombre d'exemples de cette sorte est considérable et le commentaire de Lahire sur l'assimilation ${ }^{4}$ peut sans doute bien les résumer : l'élément nouveau est toujours traité à travers les schèmes déjà existants dans nos représentations (Lahire, 2001). Si la similarité contextuelle, servant en un sens de déclencheur, ramène l'enseignant à des situations anciennes permettant d'interpréter la psychologie des apprenants, l'enseignant identifie son public à lui-même, à cette époque-là. Ainsi, la fabrique de l'action enseignante est non seulement historique, mais constitue aussi un vaet-vient entre le passé et le présent, entre le moi-apprenant de l'époque, le moienseignant actuel et le moi-public potentiel. 
19 Si les pratiques d'un enseignant peuvent prendre leur source dans divers plans de son vécu, en avançant davantage dans cette logique, on peut se demander si, en convoquant le vécu, l'enseignant ne fait pas, en quelque sorte, une mise en scène bricolée et remaniée des extraits dont le souvenir est fort?

Pour résumer, avec ou sans formation à l'enseignement, l'enseignant arrive dans une institution avec des convictions d'enseignement/apprentissage déjà plus ou moins construites. Et les principes d'enseignement reçus dans la jeunesse importent au point de jouer un rôle de socle qui peut rester intact et refuser d'évoluer. Comme dit encore Lahire, dans les "situations qui viendraient contrarier trop fortement ou trop durablement leur programme de socialisation incorporé", les enseignants peuvent rejeter l'accommodation pour mettre en place un mécanisme de défense, une sorte d'antifabrique (Lahire, 2001: 82). Cela dit, cette anti-fabrique reste très souvent au niveau cognitif :

\section{$\{00: 30: 11\} 0092$ Enseignante}

finalement le problème de de de la compréhension qu'on a eu c'est pas du tout sur euh + la création du cours ni sur l'animation mais c'est VRAIMENT sur l'évaluation qu'on était pas d'accord quoi + je lui ai dit Nathalie vous savez très bien vous avez été professeur + la simulation globale ben ne va pas s'évaluer de la même façon qu'une méthode communicative $\downarrow+$ ainsi de suite donc: pourquoi euh OBLIGER alors qu'il y a pas d'obligation de de de progression tout cela et de manuels et de cours $\uparrow+$ POURQUOI OBLIGER forcément l'examen final $\uparrow$

21 L'enseignante française est, au fond, en désaccord avec le mode d'évaluation imposé par l'institution, mais elle finit par l'appliquer. Ces deux enseignants, au moment où leurs choix méthodologiques divergent avec la méthodologie préconisée par l'institution ou par des collègues expérimentés avec lesquels ils font partie du même programme, verbalisent très clairement pendant l'entretien la pertinence de leur propre choix, même si ce n'est pas ce dernier qui a réellement été mis en place.

\section{La co-fabrique de l'action enseignante par l'enseignant, les apprenants et le contexte}

22 Le rôle de l'institution dans la construction des pratiques enseignantes étant révélé (cf. supra), l'action enseignante ne peut évoluer sans éléments extérieurs. Même si l'enseignant est maitre de sa propre pensée et de ses propres actions, en situation didactique, l'action de l'enseignant est, dans la plupart des cas, située dans un cadre sociohistoriquement déterminé, orientée vers un public donné et observée par l'institution. Les convictions de l'enseignant se trouvent ainsi en interaction constante avec les représentations des apprenants, les répertoires didactiques de ses collègues et les contraintes institutionnelles, explicites ou implicites. Le contact avec ces éléments produirait sans doute non seulement un impact sur les pratiques de l'enseignant, mais aussi sur les comportements des autres interactants. Nous proposons de nommer ici ce va-et-vient entre l'enseignant et les autres protagonistes ou facteurs contextuels la cofabrique de l'action enseignante.

23 Toujours par rapport aux deux enseignants participants, à la fin du semestre, l'enseignante française, au lieu de passer vingt minutes de plus que prévu à reformuler 
ses consignes comme au début du semestre, préfère expliquer en anglais quand les apprenants ne comprennent pas :

\title{
1er entretien d'auto-confrontation \{00:26:05\} 0211 Enseignante
}

\author{
je pense que j'ai (soupir) j'ai perdu du temps + bon en même temps c'est \\ bien qu'ils posent des questions + je j'explique mais j'aurais j'aurais dû faire \\ quelque chose de plus clair + quand même je je j'ai gaspillé 15 minutes + on \\ peut pas passer 15 minutes sur une consigne
}

\section{2e entretien d'auto-confrontation $\{00: 55: 03\} 0368$ Enseignante}

[...] et là je suis passée en anglais parce que j'ai vu que : ++ que voilà c'était compliqué $\uparrow$ et je voulais pas passer trop de temps à expliquer la consigne

On peut constater qu'au début du semestre, même si, selon l'enseignante, il n'est pas pertinent de passer quinze minutes à présenter une consigne, dans la scène commentée, ce principe didactique n'est pas mis en place. Cependant, à la fin du semestre, face à une situation similaire, donc à la réception non satisfaisante de la consigne de la part du public, pourquoi l'enseignante a-t-elle pu, cette fois-ci, prendre en considération ce principe dans la réalisation de son action? Deux hypothèses peuvent sans doute l'expliquer. Il se peut que l'enseignante débutante, face à l'obstacle, plus précisément face à un public ayant des difficultés à comprendre sa consigne, n'ait pas trouvé le moyen d'appliquer ce principe, autrement dit, de trouver une meilleure solution que la reformulation de la consigne. L'expression "quelque chose de plus clair" en est la preuve : elle avait besoin d'un moyen plus adapté de transmettre la consigne, mais la caractéristique indéfinie et indéterminée de "quelque chose" prouve que l'enseignante était encore en phase de recherche de ce moyen. L'autre possibilité est liée à la nature de la réflexion-dans-l'action (Schön, 1983), type de réflexion rapide qui

[...] guide un processus de décision, sans recours possible à des avis externes, sans possibilité de demander un "temps mort", comme une équipe de basket-ball a le droit de le faire durant un match. (Perrenoud, 1998 : n.p.).

En situation didactique, l'enseignant est souvent obligé d'agir dans l'urgence en prenant en compte des éléments de dimensions diverses. Impossible d'avoir accès à l'intake de l'enseignante française au moment de l'action, on ne peut avoir que sa réflexion-surl'action, donc ses auto-commentaires sur la scène filmée revécue au moment de l'entretien. Toujours dans ce premier extrait, le principe didactique de "ne pas passer quinze minutes sur une consigne" est prononcé par l'enseignante comme un argument justifiant la non-pertinence de son choix. Au moment de l'action, toute son attention étant focalisée sur les étudiants, elle ne peut la consacrer à d'autres éléments. Ainsi, ce principe n'est probablement parvenu/revenu à la conscience de l'enseignante qu' $a$ posteriori, qu'au moment où elle termine l'action en remarquant la gestion du temps déplanifiée. Ce point peut d'ailleurs être justifié par le discours de l'enseignante lors d'un autre type d'entretien mis en place, l'entretien post-séance visant à obtenir le commentaire de l'enseignante à chaud, c'est-à-dire juste après une séance.

Néanmoins, quand on regarde le deuxième extrait, à la fin du semestre, non seulement l'enseignante a trouvé le moyen - le passage par l'anglais - de faciliter l'accès à sa consigne aux étudiants en difficulté, mais, lors du cours, le principe didactique est cette fois intégré dans la réalisation de ses pratiques.

Cette évolution a donc un rapport intime avec le public en question. C'est en interprétant la psychologie et la réaction du public que l'enseignante commence à réfléchir à ses 
pratiques. Si, au départ, c'est la difficulté des apprenants vis-à-vis de la compréhension de ses consignes qui la motive, quitte à abandonner sa planification, à les reformuler davantage, à la fin du semestre, c'est toujours en tenant compte du profil des apprenants sinophones qu'elle adopte l'anglais comme langue intermédiaire dans les circonstances où elle rencontre des difficultés pour la transmission des consignes. Cette évolution significative révèle le côté mouvementé des pratiques enseignantes d'une enseignante novice. Il ne s'agit pas ici d'un cas où l'enseignante construit un nouveau principe didactique, mais plutôt de la mise en place d'un principe déjà existant dans des circonstances concrètes pouvant d'ailleurs empêcher sa réalisation.

Quant à l'enseignant sinophone, des phénomènes similaires peuvent être remarqués dans son discours :

\section{$\{00: 26: 03\} 0128$ Enseignant}

T'as vu t'as vu + tant que j'écris au tableau + tout le monde est actif + nerveux + c'est-à-dire on peut pas ne pas écrire + comment dire + je veux pas écrire mais en même temps je peux pas ne pas écrire + parce que sinon + ils auront l'air de n'avoir aucune idée de ce que tu disais + je ne sais pas comment faire

$\{00: 26: 26\} 0130$ Enseignant

[...] on a vraiment pas besoin de noter + si on note + c'est pour se rassurer (rire de l'enquêtrice) + c'est-à-dire aujourd'hui j'apporterai quelque chose chez moi quoi + oui $+[. .$.

\section{$\{00: 28: 24\} 0136$ Enseignant}

Donc elle elle a dit qu'elle voudrait cette place pour bien voir le tableau + mais elle a déjà compris + mais elle veut toujours ça + en fait elle est une élève très studieuse + elle arrive à comprendre + mais elle n'a toujours pas l'esprit tranquille + elle veut encore noter + même tout ça elle a déjà appris dans le cours de grammaire + elle note toujours + c'est juste pour se rassurer + en fait je pense que c'est les étudiants français + bon je peux pas dire ça car j'ai pas enseigné aux étudiants des autres pays.

L'enseignant de chinois désapprouve l'écrit dans son cours de conversation mais laisse à chaque séance un tableau rempli dans le seul but de "rassurer ses étudiants français qui adorent écrire". Si l'enseignante française a pu trouver un moyen pour satisfaire aussi bien son principe didactique que la demande du public, l'enseignant sinophone a dû complètement abandonner son principe face au public en question : envisageant comme cours de conversation "idéal" un cours sans écrit, il y renonce en prenant en compte l'habitude d'apprentissage de son public. Nous n'avons pas besoin d'entrer dans des discussions détaillées pour savoir si la prise de notes fait vraiment partie des habitudes d'apprentissage du public français, car c'est au fond en fonction de ses interprétations de la psychologie du public, donc de ses propres représentations, que l'enseignant modifie ses pratiques. Le rôle des apprenants dans la construction des pratiques enseignantes apparait clairement. Nous pouvons d'ailleurs imaginer que l'image de cet enseignant chinois varie en fonction des groupes car ses critères d'évaluation dépendent beaucoup de la matière et du public en question.

Ce qui est commun chez ces deux enseignants, c'est que la fabrique de leur agir professoral est toujours contextualisée. Face à un groupe d'apprenants, ils mobilisent tout leur répertoire didactique en modifiant ou contrôlant les éléments qui peuvent être 
inappropriés pour le cas en question et en y intégrant des techniques nouvelles qui attendent d'être testées. Il s'agit ainsi d'un processus durant lequel le système du répertoire didactique d'un enseignant, avec l'intervention du public et du contexte, se spécifie et crée des sous-systèmes "contextualisés". D'où la catégorisation des apprenants dans la pensée enseignante ainsi que celle des méthodologies correspondantes. Pendant les entretiens, l'enseignant parle non seulement des apprenants de tel groupe ou de tel pays, mais également de ce qu'il a employé ou pourrait éventuellement mettre en place comme méthodologie avec cette catégorie de public. Le côté à la fois éphémère et stable de l'action enseignante peut ainsi être révélé, on y constate :

- des actions routinières et des principes d'enseignement consolidés et conducteurs;

- des techniques qui ne s'emploient qu'occasionnellement avec un public donné et qui n'arrêtent pas de s'enrichir avec la rencontre de nouvelles classes.

Par conséquent, l'action enseignante est un résultat de co-fabrique entre l'enseignant et l'apprenant: l'un interprète à sa propre manière la pensée et l'action de l'autre pour ensuite modifier et adapter son comportement. D'ailleurs, en ce sens, il s'agit non seulement d'une co-fabrique de l'action enseignante, mais aussi de celle de l'action apprenante. Ces co-fabriques importent dans le sens où un équilibre doit être maintenu entre les efforts consacrés par les participants des deux bords. L'excès comme l'insuffisance peuvent provoquer des problèmes.

Dans cette co-fabrique de l'action enseignante ou apprenante interviennent également des éléments contextuels tels que l'institution, comme ce qui est montré dans la partie précédente (cf. supra). La charge de travail, les relations entre les collègues ainsi que la rencontre de différentes cultures éducatives peuvent exercer une influence remarquable sur l'action enseignante et la faire évoluer. Il serait ainsi intéressant d'analyser l'action enseignante à partir des schémas actionnels comme par exemple la théorie de l'activité (Engeström, Miettinen, Punamäki, 1999).

\section{La re-fabrique de l'action enseignante: une discussion méthodologique et épistémologique}

Au plan méthodologique, Schütz nous apprend que

c'est seulement dans la mesure où l'acteur fait retour sur son passé et devient ainsi un observateur de ses propres actes, qu'il peut réussir à saisir les véritables motifs parce-que de ses propres actes (1998: 58).

Et le dispositif de l'EAC, composé d'un travail d'observation et d'enregistrement vidéoscopique, fournit effectivement à l'acteur les conditions nécessaires pour un retour sur ses actes. Cela dit, la verbalisation de l'enseignant à propos de ses actions nous expliquerait-elle ses motifs véritables? Nous tenterons plutôt de considérer les autocommentaires de l'enseignant formulés à l'occasion de l'EAC comme une réinterprétation des actions qui ont eu lieu, un revécu de l'expérience avec un regard distancié, donc une re-fabrique de l'action enseignante.

L'expérience montre que la mémoire de l'enseignant peut être tout de suite activée par la scène de la classe filmée, néanmoins, les verbalisations se concentrent rarement strictement sur l'épisode en question :

$\{00: 40: 09\} 0293$ Enseignante 
[...] je pense que : ça dépend des publics là + peut-être + cet été j'ai enseigné à des Portugais $\uparrow+$ et eux ils prenaient la parole même sans lever les doigts + ils parlent ils racontent leur vie (rire) + même si c'est pas intéressant + enfin bon + euh + que j'avais pas forcément besoin de désigner + là pour cette classe $\uparrow$ comme ils sont peut-être beaucoup $\uparrow+$ ils veulent pas trop parler $\uparrow$ + je suis obligée de désigner pour obtenir un peu de réponse + (rire)

\section{$\{00: 05: 23\} 0045$ Enseignant}

[...] on se rencontre souvent à la fac les enseignants de licence + parce que nous sommes tous les enseignants du département donc on se voit souvent + [...] mais les enseignants du cours du soir certains d'entre eux sont des enseignants extérieurs + donc on a pas souvent l'occasion de se voir [...]

L'enseignante française, en interprétant le comportement de ses étudiants sinophones, se met à parler des étudiants portugais qu'elle a eus ; l'enseignant sinophone sort aussi du cours en question, cours du soir d'un diplôme universitaire, pour parler de ses collègues du programme de licence. Pendant l'EAC, l'enseignant peut commencer, au bout d'un moment, à raconter ce qui s'est passé avec un autre groupe d'apprenants, à donner un discours biographique sur un apprenant ou à parler d'un collègue que l'enquêtrice n'a jamais rencontré.

Ces informations, apparemment "digressives", possèdent une valeur non négligeable et prouvent surtout qu'en donnant du sens à ses actions, l'enseignant active tout son répertoire didactique. "Le présent est vu, perçu, interprété à travers nos abrégés d'expériences passées [...]" (Lahire, $2001:$ 101).

Si, pendant l'EAC, comme nous l'avons indiqué dans la partie précédente, la verbalisation de l'enseignant relève plutôt d'une réflexion-sur-l'action mais pas de sa réflexion-dansl'action, qui est d'ailleurs quasi impossible à reconstruire, le dispositif même de l'EAC n'est-il pas, en un sens, en train de donner à l'enseignant des conditions favorables à la réflexion? Il s'agit de ce que Theureau nomme le paradoxe de l'autoconfrontation :

l'acteur est mis, grâce à l'enregistrement vidéo, en position de développer sa réflexion située sur son activité, et il lui est demandé, dans l'autoconfrontation proprement dite de ne pas faire usage de cette possibilité, mais de se contenter d'exprimer sa conscience préréflexive au moment de la réalisation de son activité. (2010:306).

Si, dans la verbalisation de l'enseignant, des indicateurs du temps verbal tels que "je voulais" peuvent donner des pistes sur ses motifs au moment de l'action, des "j'aurais dû" et tout ce qui relève du présent sont plutôt des réflexions et des interprétations réalisées au moment de l'entretien ou, au moins, après l'action. De ce fait, la fonction de l'EAC dans les recherches sur la pensée enseignante serait-elle à redéfinir?

Dans Pensée et langage, en retraçant la relation entre langage et pensée d'un point de vue développemental et ontogénétique, Vygotski a révélé que la relation entre la pensée et la parole "apparaît, se modifie et prend de l'importance au cours de leur développement même." (1997 : 416). C'est-à-dire qu'entre la parole et la pensée, génétiquement sans relation, se nouent des liens à partir d'un moment critique où "le langage devient intellectuel, la pensée devient verbale" chez l'enfant (1997 : 182). La pensée verbale implique en premier lieu le rôle du langage dans la construction de la pensée : c'est à l'aide du mot que les êtres humains arrivent à "symboliser le concept abstrait et [à] l'utiliser en tant que signe supérieur 
entre tous ceux qu'a créés la pensée humaine." (1997 : 258). Bref, l'action même de mise en mot possède comme fonction d'organiser et de faire développer la pensée.

La pensée verbale ne se développe pas toute seule. Les éléments extérieurs, l'environnement socioculturel, avec des traces historiques consolidées, sont à la fois stimuli et sources de données et d'étayage. Ainsi, "la pensée verbale, est non pas une forme naturelle de comportement mais une forme socio-historique" (1997 : 187). De ce fait, le discours, la pensée et le comportement d'un individu sont socio-historico-culturellement marqués. Sur ces deux points, Bakhtine rejoint Vygotski :

[...] ma pensée ne saurait être définitivement claire avant que je ne lui aie trouvé une formulation verbale précise et que je ne l'aie confrontée aux propositions scientifiques touchant le même objet [...] (Bakhtine, $1980: 186)$.

En critiquant l'idée de Saussure (1967) qui considère que la langue est sociale et la parole individuelle, Bakhtine souligne que la parole est également un acte socialisé dans le sens où l'individu parle toujours en se référant à "un stock d'énoncés attendus, prototypes des manières de dire ou de ne pas dire dans un espace sociodiscursif" qu'il nomme genre (Clot, 2002 : 195). D'ailleurs, "s'il nous fallait créer pour la première fois dans l'échange chacun de nos énoncés, cet échange serait impossible." (Bakhtine, 1984 : 285).

Si le discours d'un individu est toujours un produit sociohistorique, rien n'est étonnant à ce que l'enseignant, pendant l'EAC, parle d'éléments autres que ceux qui se trouvent dans l'extrait filmé. Dans ses flux de pensée, tous ces éléments interagissent et constituent une entité, un ensemble, donc un répertoire didactique. Tout élément aura la possibilité d'émerger quand un élément contextuel le permet (Varela, 1996). Ainsi, bien que l'EAC relève en partie de la réflexion de l'enseignant a posteriori sur ses actions, avec des images vidéoscopiques comme stimuli et contrôles, des traces "historiques" de sa pensée au moment du cours vont toujours transparaitre.

Le "paradoxe d'autoconfrontation" est ainsi inévitable : l'accès à la pensée enseignante ne sera pas possible sans la mise en place du dispositif. Il est cependant également naturel : comme nous l'avons vu plus haut, dès que la mise en mots de la pensée se déclenche, elle a la possibilité de faciliter la prise de conscience et d'influencer l'organisation de la pensée. Ainsi, quelle que soit la nature de l'entretien et du discours, ce phénomène sera toujours présent. N'oublions pas non plus que sans présence du dispositif de l'EAC, l'enseignant sera toujours dans une réflexion constante, ce qui est naturel et normal dans ce métier. Si chaque enseignant, en sortant de sa classe, s'auto-évalue et s'auto-critique, c'est parce que l'agir professoral ne se limite jamais à la classe de langue. Comme souligne Cicurel : "pour accomplir son métier d'enseignant, le professeur exécute une suite d'actions en général coordonnées [...] avec une certaine intentionnalité." (2011: 119). Et le métier d'enseignant est parmi les plus concernés par la réflexivité et la réflexion (Perrenoud, 2001).

Cela ne signifie cependant pas que par l'EAC, tous les motifs qui se trouvent derrière l'action de l'enseignant vont être présentés tels quels dans sa verbalisation. Bien au contraire, le côté sociohistorique du discours de l'enseignant implique toutes les nécessités de la comprendre comme une interprétation. Et Vygostki signale aussi que la pensée verbale "n'épuise ni toutes les formes de la pensée, ni toutes les formes du langage." (1997 : 177). Dans l'action enseignante, il existe des éléments relevant d'autres dimensions qui ne peuvent pas être verbalisés. 
47 Ainsi, le discours de l'enseignant issu de l'EAC ne permet pas une vision complète des actions de l'enseignant, mais plutôt des pistes d'interprétation. En ce sens, il sera sans doute plus prudent de considérer la verbalisation de l'enseignant comme une re-fabrique de l'action déjà réellement fabriquée. Cette re-fabrique de l'action peut être comprise dans un sens encore plus large, c'est-à-dire que les réflexions constantes de l'enseignant sur ses cours réalisés, tous ses commentaires, questionnements et analyses, motivés par l'initiative professionnelle ou l'émotion, se réalisent par et dans un revécu de ses actions. C'est par ces re-fabriques de l'action enseignante que l'enseignant modifie ou consolide ses actions et ses convictions.

\section{Conclusion}

Chez les deux enseignants participants, l'action enseignante évolue : elle est un produit à la fois historique, culturel et individuel sans cesse remis en cause et actualisé. Les convictions moulées par apprenticeship of observation vont, avec la rencontre de différents publics et de différentes institutions, avec les connaissances et les expériences d'enseignement ultérieurement acquises, s'enrichir et s'affiner. L'action d'un enseignant est ainsi marquée par sa personnalité, ses expériences personnelles, les publics rencontrés et les institutions où il évolue, elle est le résultat des interactions entre ces éléments multidimensionnels. De ce fait, l'enseignant est dans des questionnements et des réflexions constants sur l'équilibre entre ses convictions didactiques, la perception du public et la demande institutionnelle, d'où la co-fabrique, la re-fabrique et éventuellement l' anti-fabrique de son action enseignante.

Si l'EAC peut présenter des faiblesses dans les recherches sur la pensée enseignante, il serait sans doute pertinent de faire intervenir un dispositif plus complet, par exemple avec des entretiens pré-séance et post-séance et des entretiens semi-directifs pré- et post-semestriels qui pourraient éventuellement fournir des données propres à vérifier les interprétations issues de l'EAC.

Pour conclure, n'oublions pas le rôle du chercheur dans la fabrique de l'action enseignante, car c'est lui qui, au fond, conçoit le dispositif méthodologique et interprète les données. Un travail d'équipe semble ainsi nécessaire pour contrôler le côté subjectif de l'analyse individuelle.

\section{BIBLIOGRAPHIE}

Bakhtine, M. (1980). Le Freudisme. Lausanne : L'âge d'homme.

Bakhtine, M. (1984). Esthétique de la création verbale. Paris : Gallimard.

Borg, S. (2003). "Teacher cognition in language teaching: A review of research on what language teachers think, know, believe, and do". Language Teaching, vol. 36, n 2. pp. 81-109. 
Borg, S. (2006). "The distinctive characteristics of foreign language teachers". Language Teaching Research, vol. 10, n 1. pp. 3-31.

Cicurel, F. (2002). "La classe de langue un lieu ordinaire, une interaction complexe". Aile, $\mathrm{n}^{\circ} 16$. pp. $145-164$.

Cicurel, F. (2011). Les interactions dans l'enseignement des langues : agir professoral et pratiques de classe . Paris : Didier.

Clot, Y. (2002). "De Vygotski à Léontiev via Bakhtine". In Clot, Y. (dir.). Avec Vygotski. Paris : La dispute. pp. 191-212.

Clot, Y. et al. (2001). "Entretiens en autoconfrontation croisée : une méthode en clinique de l'activité". Éducation permanente, $\mathrm{n}^{\circ} 146$. pp. 17-25.

Engeström, Y., Miettinen, R. \& Punamäki, R.L. (1999). Perspectives on Activity Theory. New York : Cambridge University Press.

Lahire, B. (2001). L'homme pluriel. Les ressorts de l'action. Paris : Armand Colin.

Lortie, D. (1975). Schoolteacher. Chicago : University of Chicago Press.

Maingueneau, D. (1994). L'énonciation en linguistique française. Paris : Hachette Supérieur.

Maingueneau, D. (2009). Les termes clés de l'analyse du discours. Paris : Hachette Supérieur.

Mann, S. (2005). "The language teacher's development". Language Teaching, n 38. pp. 103-118.

Mortureux, M.-F. (1993). "Paradigmes désignationnels". Semen, nº 8. pp. 123-141.

Perrenoud, Ph. (1998). "De la réflexion dans le feu de l'action à une pratique réflexive".

Disponible en ligne. www.unige.ch/fapse/SSE/teachers/perrenoud/php_main/

php_1998/1998_31.rtf.

Perrenoud, Ph. (2001). "De la pratique réflexive au travail sur l'habitus". Recherche et Formation, $\mathrm{n}$ -35. pp. 131-162.

Piaget, J. (1974). Adaptation vitale et psychologie de l'intelligence : sélection organique et phénocopie. Paris : Hermann.

Saussure, F. de. (1967). Cours de linguistique générale, 1er fasc. : [Introduction]. (R. Engler, Ed.). Wiesbaden : O. Harrassowitz.

Schön, D. (1983). The Reflective Practitioner, How Professionals Think in Action. New York : Basic Books ; traduction française par Heynemand, J. \& Gagnon, D. (1994). Le praticien réflexif, à la recherche du savoir caché dans l'agir professionnel. Montréal : Les Éditions Logiques.

Schütz, A. (1998). Éléments de sociologie phénoménologique. Paris : L’Harmattan.

Theureau, J. (2010). "Les entretiens d'autoconfrontation et de remise en situation par les traces matérielles et le programme de recherche "cours d'action"". Revue d'anthropologie des connaissances, vol. $4, \mathrm{n}^{\circ} 2$. pp. 287-322.

Varela, F. J. (1996). Quel savoir pour l'éthique? Action, sagesse et cognition. Paris : Éditions la découverte.

Vygotski, L. ([1985] 1997). Pensée et langage (F. Sève, trad.). Paris : La Dispute. 


\title{
ANNEXES
}

\section{Conventions de transcription}

La transcription des données est faite à l'aide du logiciel Folker selon les conventions de transcription suivantes :

\author{
"+" : silence \\ "++" : silence > 2 secondes \\ ":" : syllabe allongée \\ $" \uparrow "$ : intonation montante \\ $" \downarrow "$ : intonation descendante
}

"Con-ven-tion" : dit avec segmentation syllabique

"CONVENTION" : dit plus fort

"(rire)" : information additionnelle et contextuelle

"XXX" : incompréhensible

\section{NOTES}

1. Les entretiens d'auto-confrontation de ces deux enseignants sont issus d'une recherche de plus grande envergure portant sur l'évolution de la pensée enseignante des enseignants de langues. Six enseignants de français langue étrangère et de chinois langue étrangère sont suivis pendant la durée d'un semestre par un ensemble de dispositifs composé d'un entretien pré-semestriel, d'entretiens post-séance et d'entretiens d'auto-confrontation simples et croisés.

2. Les éléments modaux regroupent un groupe considérable : "adverbes et locutions adverbiales[..], interjections[..], adjectifs[..], verbes[..], modes du verbe[..], temps verbaux[..], gloses métadiscursives[..], décalages énonciatifs de divers ordres[..], signaux typographiques[..]..." (Maingueneau, 2009 : 88).

3. Cicurel définit le "répertoire didactique" de l'enseignant comme "un ensemble hétéroclite de modèles, de savoirs, des situations sur lesquels un enseignant s'appuie. Ce répertoire se constitue au fil des rencontres avec divers modèles didactiques [...], par la formation académique et pédagogique auquel il a été exposé, par l'expérience d'enseignement qui elle-même modifie le répertoire." (2002 : 157).

4. Piaget (1974) distingue chez l'individu deux mécanismes d'adaptation à l'environnement: assimilation - intégration des éléments nouvellement rencontrés aux schémas préexistants sans modifier ces derniers - et accommodation - transformation et modification des schémas préexistants auxquels s'opposent les éléments nouvellement rencontrés. 


\section{RÉSUMÉS}

L'action enseignante s'enrichit à travers l'itinéraire professionnel personnalisé de chaque enseignant. Si des particularités peuvent être repérées dans la manière dont chaque enseignant conçoit son métier, l'action enseignante peut, pour un même enseignant, évoluer en fonction de facteurs internes et externes. Cet article s'attache à interpréter la fabrique de l'action enseignante à partir d'un point de vue diachronique. À travers l'évolution de l'agir professoral de deux enseignants, novices, de chinois langue étrangère et de français langue étrangère, différentes formes de construction des pratiques enseignantes, synthétisées en fonction des catégories de l'anti-fabrique, la co-fabrique et la re-fabrique de l'action enseignante, sont à analyser et à mettre en relation.

Personalized career path enriches teacher's teaching practice. If the way that each teacher acts in his/her teaching activities has its own particularities, it could also evolve with internal and external factors. This article attempts to interpret the change of teacher's teaching practice from a diachronic point of view. By observing and interviewing during one semester two novice teachers - a teacher of Chinese as a foreign language and a teacher of French as a foreign language, we try to see how they view the evolution of their teaching practice. All the forms of evolution, declared by the participating teachers during interviews and categorized by terms as anti-fabrique, co-fabrique and re-fabrique of teaching practice are analyzed and discussed.

\section{INDEX}

Mots-clés : pensée enseignante, agir professoral, français langue étrangère, chinois langue étrangère

Keywords : teacher thinking, teaching practice, french as a foreign language, chinese as a foreign language

\section{AUTEUR}

\section{LIN XUE}

Université Sorbonne Nouvelle - Paris 3, Diltec, France

Lin Xue est doctorante contractuelle avec mission d'enseignement au laboratoire Diltec à l'université Sorbonne Nouvelle - Paris 3. Ses thématiques de recherche s'articulent autour de la pensée enseignante des enseignants de français langue étrangère et de chinois langue étrangère, de la culture éducative et de l'analyse du discours.

Toile : http://www.univ-paris3.fr/mme-xue-lin-232302.kjsp?RH=1310459924505.

Courriel : xuelinshirley[at]hotmail.com. 